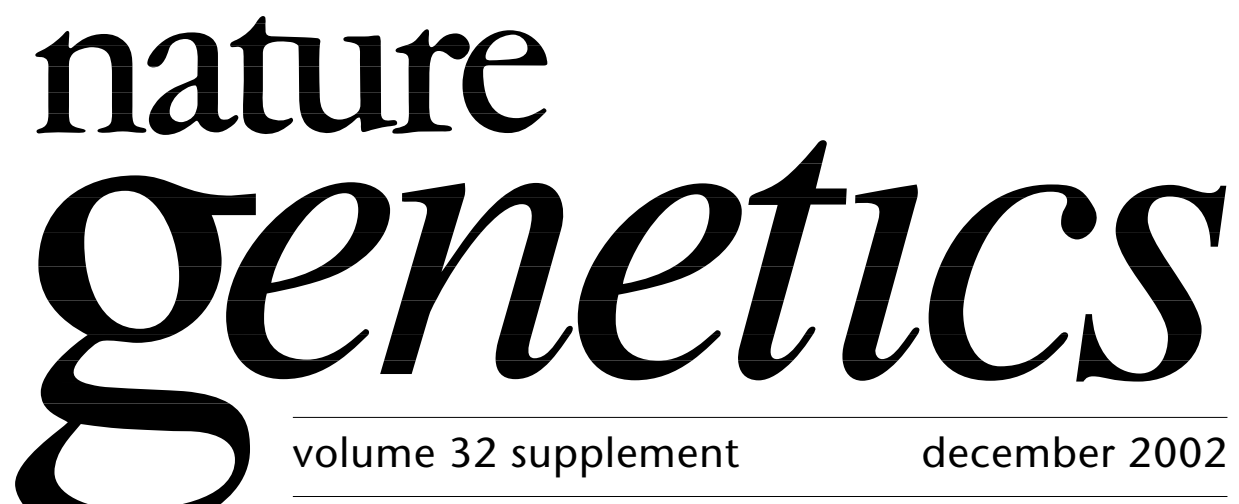

\title{
Taking stock
}

doi:10.1038/ng1024

The Chipping Forecast was published in January 1999 as a special supplement to Nature Genetics that described the 'nuts and bolts' of microarray technology. At the time, microarrays were being used in few laboratories, and articles in the supplement explained how microarrays were made, processed and used. An early recognition of the potential of microarrays by many researchers rapidly led to their adoption in laboratories throughout the world. Three years later, the use of microarrays to monitor global changes in gene expression is widespread, and an array of new developments-in hardware, methodologies and analysis-has occurred. Microarrays have made important contributions to both basic and applied research, and promise to change the practice of medicine. But, in order for their full potential to be realized as a research and diagnostic tool, many challenges must be overcome. It is time to take stock of the technology's true possibilities - and to scrutinize its limitations. We are therefore pleased to follow up on the popular Chipping Forecast with a second installment.

Several of the articles in this issue outline the major accomplishments of microarraysfrom elucidating how the genome is organized and how developmental processes are orchestrated, to aiding in the diagnosis and prognosis of cancer and in the selection of drug targets. For those entering the microarray arena, some of the biggest concerns are no longer how to make and use arrays, but rather how to design experiments, weed out noise, analyze data and validate results, and a number of articles were selected with these issues in mind. We hope that this collection of articles will provide both a practical guide that picks up where the original Chipping Forecast left off and a critical perspective of what microarrays have achieved and can achieve.

We are grateful to the National Human Genome Research Institute (NHGRI) and Agilent Technologies for generously sponsoring the publication of this volume, the contents of which will be freely available online. In addition to the authors, many individuals have provided advice at various stages of the development of this supplement. We thank Jeffrey Trent, Mark Boguski, Todd Golub, Tim Aitman, Joe Hacia, Richard Young, Jason Lieb, Cindy Afshari, Jeffrey Waring, Janet Warrington, Mel Kronick, Leonid Kruglyak, Susan Mango, Arul Chinnaiyan, Steve Madden, Daniel Geshwind, Sridhar Ramaswamy, Marc Vidal, Richard Simon, Greg Gibson, Russell Wolfinger, Lance Liotta, Joe Gray, Vito Quaranta, David Rocke, Gavin Sherlock, Erica Golemis, Steen Knudsen, Martin Hessner and others whom we have consulted. Eric D. Smith from Stanley Korsmeyer's laboratory provided the cover image. 